\title{
Lentiviral-mediated let-7d microRNA overexpression induced anxiolytic- and anti-depressant-like behaviors and impaired dopamine D3 receptor expression
}

\author{
Amine Bahi ${ }^{a, *}$, Jean-Luc Dreyer ${ }^{b}$ \\ a Department of Anatomy, Tawam Medical Campus, United Arab Emirates University, Al Ain, UAE \\ ${ }^{\mathrm{b}}$ Division of Biochemistry, Department of Medicine, University of Fribourg, $\mathrm{CH}-1700$ Fribourg, \\ Switzerland
}

\author{
KEYWORDS \\ Anxiety; \\ Depression; \\ Dopamine D3 receptor; \\ Lentiviral vector; \\ let-7d; \\ microRNAs
}

\begin{abstract}
Generalized anxiety and major depression disorders (MDD) are severe debilitating mood disorders whose etiology are not fully understood, but growing evidence indicates that microRNAs (miRNAs) might play a key role in their neuropathophysiological mechanisms. In the current study, we investigate the role of Lethal-7 (let-7d) miRNA, and its direct target dopamine D3 receptor (D3R) gain-of-function, in the hippocampus, in preclinical models of anxiety and depression in mice. For this purpose, we have constructed a lentiviral vector carrying let-7d miRNA and its anxiolytic effect was investigated by employing the open-field (OF) and the elevated plus maze (EPM) tests. The anti-depressant activity was evaluated using the tail suspension and the forced-swim tests (TST \& FST). Our results show that let-7d overexpression significantly improved the measures of anxiety in the OF and EPM tests. In addition, let-7d increased the mobility time in the TST and FST. Interestingly, gene expression interaction analysis shows that the D3R mRNA negatively correlates with let-7d expression. In a different set of experiments, we used a tetracycline-inducible (tet-off) lentiviral vector to overexpress D3R to assess its gain-of-function in the hippocampus on anxiety- and depression-like behaviors. In line, we found that in the absence of doxycycline, D3R produced a significant anxiogenic and depressant-like response. Most importantly, these effects were abrogated when mice were fed doxycycline in drinking water. Our results provide the first evidence for an anxiolytic and
\end{abstract}

Abbreviations: D3R, Dopamine D3 receptor; DOXY, Doxycycline; EPM, Elevated plus maze; FST, Forced swim test; Let-7, Lethal-7; LV, Lentiviral Vectors; miRNA, microRNA; OF, Open field; TST, Tail suspension test; WTR, Water.

* Corresponding author.

E-mail address: jean-luc.dreyer@unifr.ch (A. Bahi). 
anti-depressant-like action of let-7d through a potential D3R target-mediated mechanism which might open new avenues for anxiolytic and anti-depressant therapies.

(C) 2018 Elsevier B.V. and ECNP. All rights reserved.

\section{Introduction}

According to the National Institute of Mental Health (NIMH), and the Anxiety and Depression Association of America (ADAA), anxiety disorders are the most common mental illness in the US, affecting 40 million adults at the age 18 and older (ADAA, 2014). Although these disorders lead to significant suffering for the affected persons, their underlying causes are poorly understood. As such, identifying the molecular mechanisms common to anxiety and depression may prove crucial to successfully treating these illnesses. Previous investigations aimed at identifying the neuroadaptations pertinent to this comorbidity have revealed a potential role for microRNAs (miRNAs) dysregulations in both anxiety and depression (Giridharan et al., 2016).

MiRNAs are small non-coding RNAs that regulate protein synthesis post-transcriptionally by base-pairing to target gene mRNAs. In mammals, miRNAs have been shown as one of the key regulators that control development of the CNS, including neurogenesis (Liu and Zhao, 2009), neuronal proliferation (Cheng et al., 2005), and synaptic plasticity (Kiebler and Bassell, 2006). Since the first report identifying novel genes coding for small expressed RNAs (LagosQuintana et al., 2001), it has been shown that because miRNAs have a wide range of effects in the CNS, their implication in the pathophysiology of mood has been recognized for some time (Suarez-Gomez et al., 2011). In fact and in 2008, Uchida and co-workers reported that repeatedly restrained rats exhibited increased anxiety-related behavior that was associated to increased expression of miR-18a in the hypothalamic paraventricular nucleus (PVN) (Uchida et al., 2008). Also, molecular genetics and developmental studies have identified 21 genes in the chromosomal $8 p$ region that are most likely to contribute to neuropsychiatric disorders such as depression, with at least seven nonprotein-coding miRNAs located in the same region (Tabares-Seisdedos and Rubenstein, 2009). However, despite such a history of interest in this topic, many questions remain about the functions, brain targets, and mechanisms of miRNAs for their effects on mood disorders.

Lethal-7 (let-7) is one of the most studied miRNA families. It is the most abundant among all miRNAs and is highly conserved between species. To date, the let-7 family consists of 13 members (let-7a to let-7i, miR-98 and miR-202) located in 9 different loci on chromosomes 3, 9-12, 19, 21, 22 and $X$ (Roush and Slack, 2008). Let-7d is abundant in the brain. In fact, using a microarray screening, Shu and colleagues found that let-7d was up-regulated in the hippocampus and the Marginal division (MrD) of the neostriatum (Shu et al., 2013), suggesting that it may play an important role in modulating learning and memory (Luo et al., 2015). Also, let-7d, which is increased in the prefrontal cortex of spontaneously hypertensive rats' brain, negatively regulated galectin- 3 which was associated with attention deficit hyperactivity disorder development (Wu et al., 2010). Furthermore, let-7d regu- lated neural stem cell proliferation and promoted premature neuronal differentiation and migration (Zhao et al., 2013). In a previous study from our laboratory, we have shown a significant downregulation of the D3R following let$7 \mathrm{~d}$ overexpression in the nucleus accumbens, which was associated with altered cocaine-conditioned place preference (CPP) (Chandrasekar and Dreyer, 2011). This prompted us to investigate the potential role of let-7d and its direct target D3R in the ethiology of anxiety and depression in adult mice.

Dopamine, a major neurotransmitter in the CNS, is one of several neurotransmitters implicated in mood disorders. Dopamine exerts its effects through binding and activation of five subtypes of receptors (D1, D2, D3, D4 and D5 receptors), classified as D1-class receptors (D1 and D5) or D2-class receptors (D2, D3 and D4) (Beaulieu et al., 2015). The D3R was cloned almost three decades ago, and evidence suggested that it is expressed in the limbic areas of the brain associated with emotion and psychiatric disorders (Sokoloff et al., 1990). In fact, dysregulation of the D3R has been linked to many psychiatric diseases including schizophrenia, addiction, anxiety, depression (Leggio et al., 2016). Overall, most of the studies linking the D3R to anxiety- and depression-like behaviors in rodents, involved systemic injection of D3R pharmacological agents or transgenic mice. Therefore, we have decided to employ a technique of viralmediated gene transfer to manipulate D3R expression with a view of studying the effects of ectopic D3R mRNA levels on emotion-related behaviors. This technique had been extensively used in our laboratory to manipulate this receptor in the rat brain (Bahi et al., 2005; Bahi and Dreyer, 2014).

For this purpose, in the present study we aimed to determine the effects of viral-mediated let-7d overexpression in the hippocampus in anxiety- and depression like phenotypes in mice, by means of a battery of behavioral tests. We also assessed the expression levels of let-7d and its direct target D3R in the hippocampus following let-7d overexpression. Importantly, to confirm a potential effect of hippocampal D3R-dependent behavioral tasks, consequences of lentiviral-mediated D3R manipulation of hippocampal area on anxiety and depression tasks were also examined.

\section{Experimental procedures}

\subsection{Animals}

Adult male C57BL/ 6 mice weighing approximately $25 \mathrm{~g}$ were housed in standard Plexiglas observation cages. All mice were bred in the local central animal facility of the College of Medicine \& Health Sciences and were kept under standard laboratory conditions used to reduce mouse stress $(12 \mathrm{~h}$ light $/ 12 \mathrm{~h}$ dark cycle, lights off at $6 \mathrm{pm}$, and approximately $22{ }^{\circ} \mathrm{C}$ with $40-60 \%$ humidity). Bedding was autoclaved before use and mice had free access to tap water and stan- 


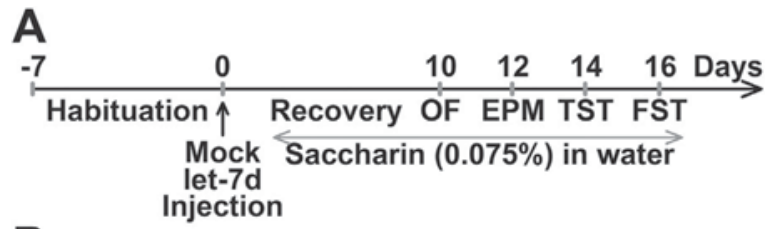

B

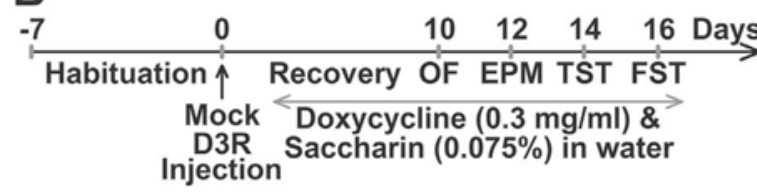

Fig. 1 Experimental timeline. The timelines show sequence and duration of experimental protocols of the effects of $A$ ) let7d, and B) D3R overexpression on anxiety- and depression-like behaviors in adult mice.

dard mouse chow diet obtained from the National Feed and Flour Production and Marketing Company LLC (Abu Dhabi, $\mathrm{UAE}$ ). The experimental procedures were approved by the local Animal Research and Ethics Committee (Protocol No A17-12).

\subsection{Construction, production of the lentiviral vectors and stereotaxic injection}

The let-7d lentiviral vectors, driven by an RNA Pol III mouse U6 promoter, were prepared as described previously (Chandrasekar and Dreyer, 2011). For the D3R-overexpressing lentiviral vectors, driven by a cytomegalovirus (CMV) promoter, the rat D3R was amplified from total brain cDNA using specific primers and ligated into pTK431 as described previously (Bahi and Dreyer, 2014).

To deliver the viruses into the hippocampus, we used stereotaxic injections as previously described for mice and rats (Bahi et al., 2014b; Bahi and Dreyer, 2017). The complete dorsal and ventral hippocampus was targeted (including the CA fields, dentate gyrus, and subiculum) at four stereotaxic coordinates: 1st injection (AP - 1.7, ML $+/-1.2$, DV - 1.5); 2nd injection (AP - 2.3, ML + /-1.75, DV - 1.75); 3rd injection (AP - 2.8, ML + / -3, DV - 3); 4th injection (AP -3.4, ML + / - 3, DV - 3.75) (Devito et al., 2010; Franklin and Paxinos, 1996). In sham-operated controls, infusions were made in the same coordinates, except that instead it was the empty PTK431 cloning vector (Mock) that was injected through the Hamilton micro-syringe. For more details, see the Supplementary Material.

In the first experiment and after surgery, the mice of each group were single-housed and left to recover for 10 days as depicted in the experiment timeline (Fig. 1A). The mice had access to $0.075 \%$ saccharin dissolved in tap water $(w / v)$. Two mice were removed from the study because they did not recover from the surgery. Therefore, for both Mock and let$7 d$ groups $n=12$ each.

In the second experiment, after surgery the mice (Mock and D3R; $n=20$ each) had access to $0.075 \%$ saccharin with or without $0.3 \mathrm{mg} / \mathrm{ml}$ doxycycline dissolved in tap water $(w / v)$ (Fig. 1B). This combination of viral-injection and doxycycline availability created four test groups $(n=10$ each): Mock-WTR, D3R-WTR, Mock-DOXY, and D3R-DOXY.

\subsection{Behavioral analyses}

All the behavioral tests were conducted between $10 \mathrm{am}$ and $4 \mathrm{pm}$, and the mice were habituated to the room for at least $1 \mathrm{~h}$ before testing. The general design of the study is depicted in Fig. $1 \mathrm{~A}$.

\subsubsection{Open field (OF) test}

Spontaneous and exploratory locomotor activity and anxiety were assessed in an OF test, 10 days after the stereotaxic injections, as reported in our previous studies (Bahi, 2017a; Bahi et al., 2016; Bahi et al., 2014a; Bahi et al., 2014c). The anxiogenic-like phenotype was supported by decreased time spent in the center and by increased number of fecal boli left in the arena (Bahi and Dreyer, 2017). After each session the arena was cleaned with a $70 \%$ pure ethanol solution to remove odor trails. For more details, see the Supplementary Material.

\subsubsection{Elevated-plus maze (EPM) test}

After the OF test, we assessed the effect of let-7d on anxiety-like behavior using the EPM test as we previously described (Bahi, 2017a, 2017b). For more details, see the Supplementary Material.

\subsubsection{Tail suspension test (TST)}

The TST is a common behavioral test to evaluate depressivelike behavior in animals and it was performed based as previously described (Bahi and Dreyer, 2012; Bahi et al., 2014c). For more details, see the Supplementary Material.

\subsubsection{Forced-swim test (FST)}

The FST was performed as we described previously (Bahi et al., 2014a; Bahi and Dreyer, 2012; Bahi et al., 2014c). For more details, see the Supplementary Material.

\subsection{RNA isolation and real-time qRT-PCR quantification}

After completion of the behavioral experiments, let-7d and D3R transcription levels were quantified using RT-PCR. In brief, the mice were killed by rapid decapitation, brains were quickly removed, and the entire hippocampal tissue was dissected out and frozen in liquid nitrogen then stored at $-80^{\circ} \mathrm{C}$. For more details, see the Supplementary Material.

For the qPCR, $20 \mu \mathrm{L}$ of amplification mixture with SYBRGreen Kit was used containing $4 \mu \mathrm{L}$ of CDNA and $100 \mathrm{nM}$ primers as described in our previous studies. For more details, see the Supplementary Material.

\subsection{Statistical analysis}

Statistical analysis was performed using SPSS version 16.0 for Windows. All data are expressed as the mean \pm SEM (Standard Error of Mean). For more details, see the Supplementary Material. 


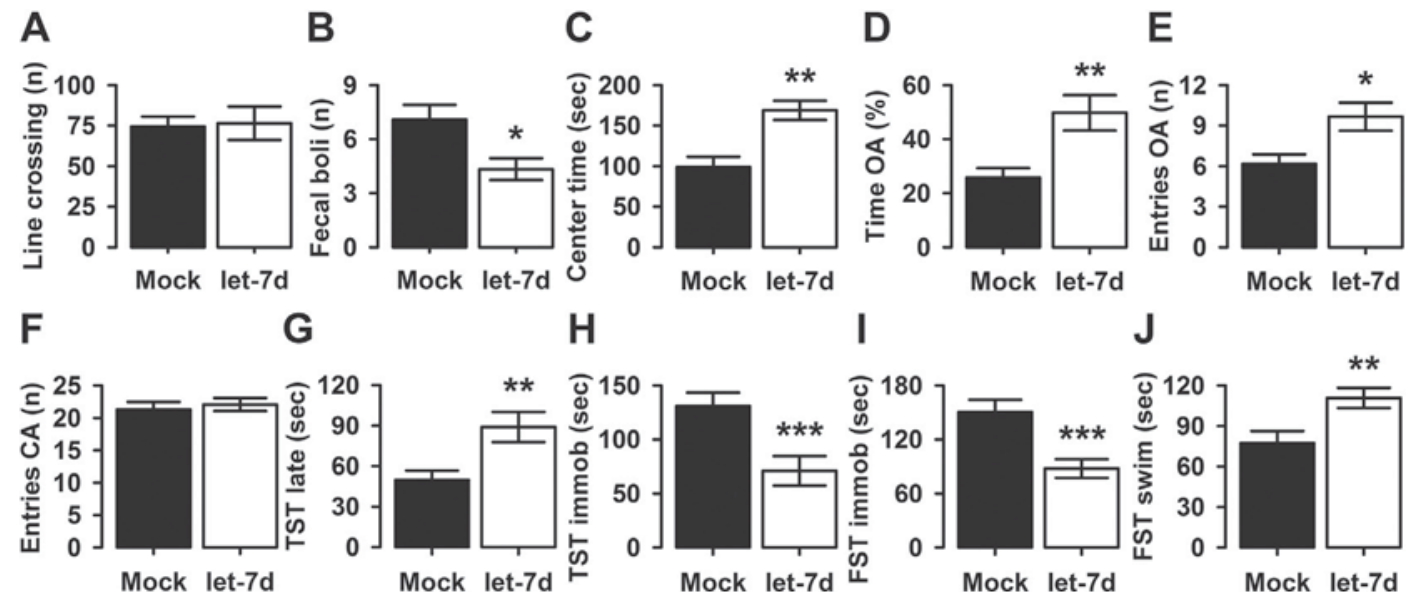

Fig. 2 Anxiety- and Depression-like behaviors like behavior in Mock- and let-7d-injected mice in the OF, EPM, TST and FST. For the OF test, the data are expressed as mean \pm SEM for the (A) number of line crossings, (B) number of fecal boli, and (C) time spent in the center of the arena. For the EPM test, the data are expressed as mean \pm SEM for the (D) percent of time spent in the open arms (OA), (E) number of entries into the OA, and F) number of entries into the closed arms (CA). For the TST, the data are expressed as mean \pm SEM for the $(\mathrm{G})$ latency, and $(\mathrm{H})$ duration of immobility. For the FST, the data are expressed as mean \pm SEM for the duration of (I) immobility, and (J) swimming. ${ }^{*} p<0.05$, ${ }^{* *} p<0.01$, and ${ }^{* * *} p<0.005$. For both groups $n=12$.

\section{Results}

The Levene's test was used to inspect the equality of variances of the groups compared and for all the analyses, the test was found to be statistically not significant (For more details, see the Suppl Tables 1 and 2).

\subsection{Effects of let-7d overexpression on anxiety-like behavior in adult mice}

The open field test (OF): this test was performed 10 days after the stereotaxic injections to investigate the effect of let-7d on anxiety-like behavior and spontaneous explorative locomotor activity. We evaluated mice performances in an OF test after injection of empty (Mock) or let-7d overexpressing lentiviral vectors $(n=12$, each). For the average distance travelled, and as depicted in Fig. 2A, the one-way ANOVA indicated that the number of line crossings did not differ significantly between the two experimental groups $\left(F_{(1,22)}=0.024, p=0.879\right)$. However, let-7d overexpression produced an anxiolytic-like response as it significantly decreased the number of fecal boli $\left(\mathrm{F}_{(1,22)}=7.256, p=0.013\right)$ (Fig. 2B), and significantly increased the time spent in the center of the arena during the OF test $\left(F_{(1,22)}=16.053\right.$, $p=0.001$ ) (Fig. 2C).

The elevated plus maze test (EPM): we also estimated anxiety behaviors in Mock and let-7d-injected mice using the EPM test. Consistent with the above results, let-7d overexpressing mice showed more time spent in $\left(F_{(1,22)}=10.486\right.$, $p=0.004)$ (Fig. 2D), and entering $\left(\mathrm{F}_{(1,22)}=7.688, p=0.011\right)$ (Levene's test: $F_{(1,22)}=0.762, p=0.392$ ) (Fig. 2E) the open arms (OA) compared with the Mock controls. However, there were no statistically significant differences between the groups in the number of entries into the closed arms (CA) $\left(F_{(1,22)}=0.245, p=0.626\right)$ (Fig. $\left.2 F\right)$. These data indicate that let-7d overexpression in the hippocampus may induce an anxiolytic-like response with no confounding locomotor activity changes.

\subsection{Effects of let-7d overexpression on depression-like behavior in adult mice}

The tail suspension test (TST): to determine whether increased hippocampal let-7d expression might be accompanied by development of an anti-depressive phenotype, we tested Mock- and let-7d-injected mice in the TST. Behavioral despair in this task is defined as an increase in immobility in response to inescapable stress and mice were considered immobile when they hung motionless with their limbs tucked against their bodies. As depicted in Fig. 2G and as compared with mice in the Mock control group, mice injected with let-7d overexpressing vectors showed a significant increase in their latency to immobility $\left(F_{(1,22)}=9.033, p=0.007\right)$. However, the duration of immobility was significantly decreased following let-7d overexpression $\left(F_{(1,22)}=10.702, p=0.003\right)$ (Fig. $\left.2 \mathrm{H}\right)$, suggesting that let-7d can reduce depression-like behavior in mice.

The forced swim test (FST): the FST was conducted after the TST, and the data analysis indicated that let-7d overexpression in the hippocampus significantly decreased the immobility time $\left(F_{(1,22)}=13.668, p=0.001\right)$, as compared with the Mock control group (Fig. 2l). However, let-7d-overexpressing mice spent significantly more time swimming $\left(F_{(1,22)}=8.219, p=0.009\right)$ (Fig. 2J), and climbing $\left(F_{(1,22)}=5.172, p=0.033\right)$ (data not shown) than their Mock controls, indicating that these mice have a significantly decreased behavioral despair and confirming the TST data, that hippocampal let-7d overexpression can reduce depression-like behavior in mice. 
A
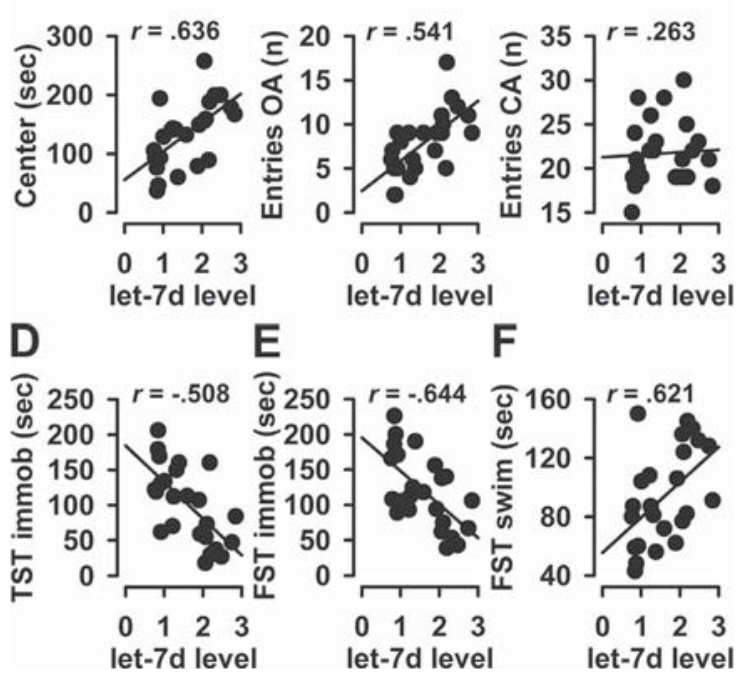

Fig. 3 Pearson's correlations between let-7d expression and anxiety- and depression like behaviors. The data represent simple scatter regression between let-7d levels with the $(A)$ time spent in the center of the arena of the OF test, (B) number of entries into the $\mathrm{OA}$, and $(\mathrm{C})$ number of entries into the $\mathrm{CA}$ of the EPM test, (D) duration of immobility in the TST, and duration of (E) immobility, (F) swimming in the FST.

\subsection{The quantification of let-7d and D3R levels using real-time $\mathrm{qRT}-\mathrm{PCR}$}

After completion of the behavioral experiments, the mice were killed by rapid decapitation, the brains were removed, the hippocampi were dissected out and let-7d levels were quantified using qRT-PCR. The quantification revealed that when let-7d was injected into the hippocampus, transcript levels increased to approximately $225 \%$ compared to Mock controls $(2.252 \pm 0.164$ and $1.000 \pm 0.160$ respectively). Thus, the one-way ANOVA analysis indicated that expression levels differed significantly between the two experimental groups $\left(F_{(1,22)}=49.054, p<0.0001\right)$.

Using the data of let-7d transcription levels obtained from the Mock- and let-7d-injected mice, a simple linear regression (Pearson's) analysis was performed to examine the correlation between let-7d transcription levels and parameters of anxiety- and depression-like behaviors and the scatter plots are provided in Fig. 3. Post hoc linear regressions revealed that differences in let-7d levels within the hippocampus accounted for many of the behavioral alterations exhibited by the mice. In fact, in the OF test, increased let$7 d$ expression was positively correlated with amount of time spent in the center of the arena $\left(F_{(1,22)}=14.954, p=0.001\right.$; Fig. 3A). Similarly, in the EPM test, the Pearson's correlation and Post-hoc corrections revealed that let-7d expression correlated positively with the number of entries into the $\mathrm{OA}$ $\left(F_{(1,22)}=9.122, p=0.006\right.$; Fig. $\left.3 B\right)$, but not with the number of entries into the $C A\left(F_{(1,22)}=1.638, p=0.214\right.$; Fig. $\left.3 C\right)$. The relations between let-7d expression levels and measures of depression were also determined. In the TST, the results have shown a significant negative correlation with immobility duration $\left(\mathrm{F}_{(1,22)}=7.665, p=0.011\right.$; Fig. 3D). Fi-
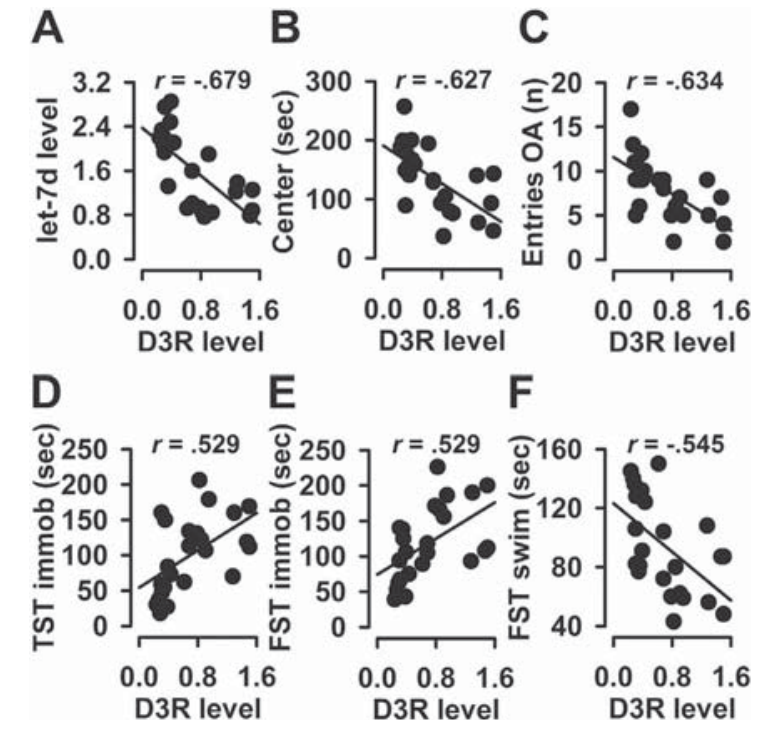

Fig. 4 Pearson's correlations between D3R mRNA expression and anxiety- and depression like behaviors. The data represent simple scatter regression between D3R levels with (A) let-7d expression. (B) time spent in the center of the arena of the OF test, (C) number of entries into the OA of the EPM test, (D) duration of immobility in the TST, and duration of (E) immobility, and $(\mathrm{F})$ swimming in the FST.

nally, in the FST, increased let-7d expression was negatively correlated with the latency to immobility $\left(F_{(1,22)}=15.600\right.$, $p=0.001$; Fig. $3 \mathrm{E})$ and positively correlated with swimming $\left(F_{(1,22)}=13.811, p=0.001\right.$; Fig. $\left.3 F\right)$. For the rest of the correlations and scatter plots see Suppl. Fig. 1.

We also quantified the mRNA levels of let-7d direct target D3R, and the expression levels in Mock- and let-7dinjected mice were $1.000 \pm 0.150$ and $0.391 \pm 0.155$ respectively. Thus, the one-way ANOVA analysis revealed that let$7 \mathrm{~d}$ overexpression resulted in an approximately $60 \%$ marked and significant down-regulation of the D3R mRNA expression in the hippocampus $\left(F_{(1,22)}=110.499, p<0.0001\right)$. Most impressively, the Pearson's analysis showed a significant negative correlation between let-7d and D3R expression levels $\left(F_{(1,22)}=18.815, p<0.0001\right.$; Fig. $\left.4 A\right)$.

Additionally, post hoc linear regressions revealed that alterations in D3R mRNA expression accounted for many of the behavioral changes. In fact, in the OF test, D3R mRNA expression correlated negatively with the time spent in the center $\left(F_{(1,22)}=14.248, p=0.001\right.$; Fig. 4B). Also, in the EPM test, D3R expression correlated negatively with the number of entries into the OA $\left(F_{(1,22)}=14.759, p=0.001\right.$; Fig. $4 \mathrm{C})$. In the TST, results also indicated a positive correlation with immobility duration $\left(\mathrm{F}_{(1,22)}=8.564, p=0.008\right.$; Fig. 4D), and finally, in the FST, decreased D3R levels were positively correlated with the immobility duration $\left(F_{(1,22)}=8.549, p=0.008\right.$; Fig. $\left.4 \mathrm{E}\right)$ and negatively correlated with swimming duration $\left(\mathrm{F}_{(1,22)}=9.280, p=0.006\right.$; Fig. $\left.4 \mathrm{~F}\right)$. For the rest of the correlations and scatter plots see Suppl. Fig. 2.

Taken together, these findings suggest that mice with high let-7d levels in the hippocampus are most likely to have low 


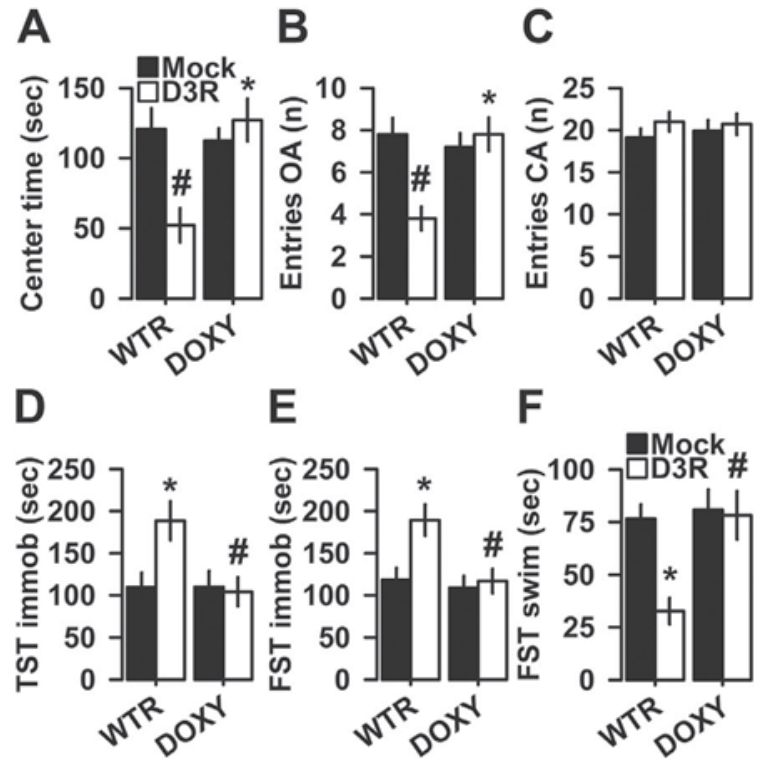

Fig. 5 Anxiety- and depression like behaviors in Mock- and D3R-injected mice in the OF, EPM, TST and FST with access to water (WTR) or doxycycline (DOX). For the OF test, the data are expressed as mean \pm SEM for the (A) time spent in the center of the arena. For the EPM test, the data are expressed as mean \pm SEM for the $(B)$ number of entries into the OA, and (C) number of entries into the CA. For the TST, the data are expressed as mean \pm SEM for the (D) duration of immobility. For the FST, the data are expressed as mean \pm SEM for the duration of (E) immobility, and (F) swimming. ${ }^{*} p<0.05$ indicate significant differences between Mock and D3R; $\# p<0.05$ indicate significant differences between WTR and DOX. For all the groups $n=10$.

D3R mRNA levels that are in turn associated with reduced anxiety- and depression-like phenotypes.

\subsection{Effects of D3R overexpression on anxiety-like behavior in adult mice}

The impact of D3R overexpression in the hippocampus on anxiety-like behavior was determined 10 and 12 days after the viral injection using the OF and the EPM tests respectively.

The open field test (OF): Fig. 5A illustrates a significantly lower time spent in the center of the arena in D3R-overexpressing mice than their Mock controls $\left(\mathrm{F}_{(1,36)}=4.218, \quad p=0.047\right)$, and the same analysis revealed that doxycycline significantly abrogated this difference $\left(F_{(1,36)}=6.513, p=0.015\right)$. Importantly, the viral-injection $\times$ doxycycline was found significant $\left(F_{(1,36)}=10.164, p=0.003\right)$. Although there was a significant effect of viral-injection across both groups, post-hoc tests revealed that this effect was driven by the WTR group (WTR-Mock vs. WTR-D3R, $p=0.004$; Bonferroni post-hoc test). In contrast, no significant difference was found in the DOX group between Mock-and D3R-injected mice $(p=1.000)$ and (WTR-D3R vs. DOX-D3R, $p=0.002$; Bonferroni post-hoc test).
The elevated plus maze test (EPM): the data from the EPM test showed that D3R-injected mice had a decreased number of entries into the OA (main effect of viral-injection: $\left.F_{(1,36)}=5.832, p=0.021\right)$, an effect that was significantly abrogated when mice had access to doxycycline $\left(F_{(1,36)}=5.832, p=0.021\right)$ with significant interaction $\left(F_{(1,36)}=10.675, p=0.002\right)$ (Fig. 5B). It should be mentioned that the D3R effects on the parameters measured in the EPM test were mainly driven by the WTR group, which differentially and significantly affected the Mock- and D3R- injected groups $(p=0.002$, Bonferroni post hoc test). Likewise, there was no significant effect of D3R overexpression on the number of entries into the OA in presence of doxycycline ( $p=1.000$ and WTR-D3R vs. DOX-D3R, $p=0.002$; Bonferroni post hoc test). The overall activity was estimated by the number of entries performed into the CA during the 5 -min observation period. And the two-way ANOVA analysis indicated that this parameter was affected following D3R overexpression $\left(F_{(1,36)}=1.282, p=0.265\right)$. Likewise, there was no significant effect of doxycycline on the number of entries into the $C A\left(F_{(1,36)}=0.044, p=0.835\right)$, nor was there a significant viral-injection by doxycycline interaction $\left(F_{(1,36)}=0.213, p=0.647\right)$ (Fig. $\left.5 C\right)$ indicating no change in overall ambulatory behavior, and suggesting that hypothetical overly sedating effects of viral injection did not confound the anxiety-like behavioral results reported above. Additional results are depicted in Suppl. Fig. 3.

\subsection{Effects of D3R overexpression on depression-like behavior in adult mice}

To investigate the effect of D3R overexpression on depression-like behavior, the TST the FST were performed.

The tail suspension test (TST): as depicted in Fig. 5D, although the effect of D3R overexpression on the duration of immobility did not reach statistical significance $\left(F_{(1,36)}=3.607, p=0.066\right)$, doxycycline blunted that effect $\left(F_{(1,36)}=4.825, p=0.035\right)$ with a significant interaction $\left(F_{(1,36)}=4.871, p=0.034\right)$. Again, the D3R effects were driven by the WTR group, which was significantly different between the viral-injection conditions $(p=0.038)$, whereas the doxycycline group did not differ between Mock- and D3R-injected rats $(p=1.000$ and WTR-D3R vs. DOX-D3R, $p=0.022$; Bonferroni post hoc test).

The forced swim test (FST): Fig. 5E showed that the interaction between viral-injection and doxycycline had a significant effect on the immobility duration $\left(F_{(1,36)}=4.215, p=0.047\right)$. The individual factors viral-injection $\left(F_{(1,36)}=6.577, p=0.015\right)$ and doxycycline $\left(F_{(1,36)}=6.982, p=0.012\right)$ also had significant effects. The D3R overexpression-induced increase of immobility was significant only in the WTR group but not in the DOXY group $(p=0.014$ and $p=1.000$ respectively) and (WTR-D3R vs. DOX-D3R, $p=0.012$; Bonferroni post hoc test). For the swimming duration and as depicted in Fig. $5 \mathrm{~F}$, both viralinjection $\left(F_{(1,36)}=6.993, p=0.012\right)$, and doxycycline treatment $\left(F_{(1,36)}=7.988, p=0.008\right)$ had significant effects, with significant interaction $\left(F_{(1,36)}=5.516, p=0.024\right)$. The effects on swimming during this test were mainly driven by the WTR group, which differentially and significantly affected the Mock- and D3R- injected groups $(p=0.007)$. However, 
there was no significant effect of D3R overexpression on swimming in presence of doxycycline $(p=1.000)$ and (WTRD3R vs. DOX-D3R, $p=0.005$; Bonferroni post hoc test). This finding indicated that doxycycline could prevent the decrease of swimming behavior following D3R overexpression. Additional results are depicted in Suppl. Fig. 4.

\subsection{Quantification of D3R levels using real-time qRT-PCR}

To investigate whether D3R overexpression and doxycycline altered the contents of D3R mRNA in the hippocampus, qRT-PCR was used. In the absence of doxycycline, D3Rinjected mice exhibited a significant increase in mRNA levels over Mock controls $(1.000 \pm 0.144$ and $1.693 \pm 0.145$ respectively). However, when doxycycline was given in drinking water, D3R mRNA quantification revealed no significant difference between Mock- and D3R experimental groups $(1.000 \pm 0.181$ and $1.138 \pm 0.129$ respectively). The two-way ANOVA analysis revealed main effect of viral-injection $\left(\mathrm{F}_{(1,36)}=57.716, p<0.0001\right)$, and doxycycline $\left(\mathrm{F}_{(1,36)}=12.265, p=0.001\right)$, with a significant interaction between the two factors $\left(F_{(1,36)}=15.066, p<0.001\right)$. Post hoc evaluations indicated that in the WTR group, D3R overexpression increased D3R mRNA levels by approximately $170 \%$ (WTR-Mock vs. WTR-D3R, $p<0.0001$ ). However, when mice had access to doxycycline in the drinking water, no significant difference was found between the two experimental groups (DOX-Mock vs. DOX-D3R, $p=0.075$ and WTR-D3R vs. DOX-D3R, $p<0.001$ ).

Using the data of D3R mRNA levels obtained from the four experimental groups, we performed a Pearson's linear regression analysis to examine the correlation between D3R mRNA levels and anxiety- and depression-like behaviors. The scatter plots are provided in Fig. 6. Post hoc linear regressions revealed that differences in D3R levels were associated with changes of behavior. In the OF test, D3R correlated negatively with the time spent in the center of the arena $\left(F_{(1,38)}=10.185, p=0.003\right.$; Fig. $\left.6 \mathrm{~A}\right)$. In the EPM test, D3R expression correlated negatively with the number of entries into the $O A\left(F_{(1,38)}=13.060, p=0.001\right.$; Fig. $\left.6 \mathrm{~B}\right)$, but not with the number of entries into the $C A\left(F_{(1,38)}=2.322\right.$, $p=0.136$; Fig. $6 \mathrm{C}$ ). In the TST, results also indicated a positive correlation with immobility duration $\left(F_{(1,38)}=18.738\right.$, $p<0.001$; Fig. 6D). Finally, in the FST, decreased D3R levels were positively correlated with the latency to immobility $\left(F_{(1,38)}=10.756, p=0.002\right.$; Fig. $\left.6 \mathrm{H}\right)$ and negatively correlated with swimming duration $\left(\mathrm{F}_{(1,38)}=12.671, p=0.001\right.$; Fig. 6F). For the rest of the correlations and scatter plots see Suppl. Fig. 5.

Taken together, these findings suggest that high D3R mRNA levels in the hippocampus are most likely to be associated with increased anxiety- and depression-like phenotypes.

\section{Discussion}

The results of the present series of experiments show, for the first time, that adult mice overexpressing let-7d in the hippocampus display a behavioral profile that is consistent

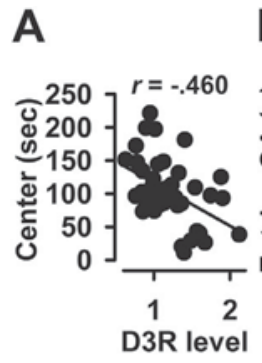

B

C

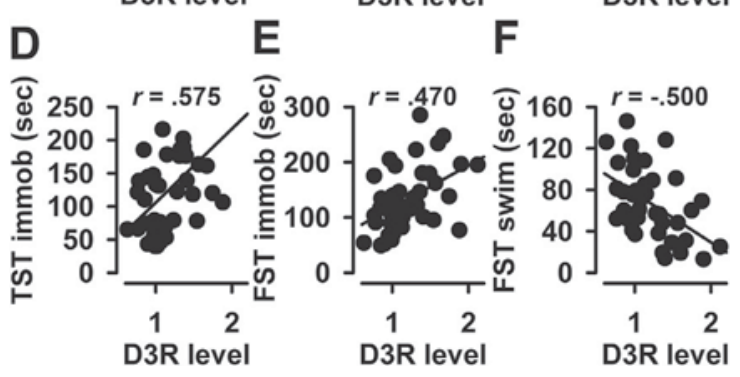

Fig. 6 Pearson's correlations between D3R mRNA expression in Mock- and D3R-injected mice and anxiety- and depression like behaviors. The data represent simple scatter regression between D3R levels with (A) time spent in the center of the arena of the OF test, $(B)$ number of entries into the $O A$, and $(C)$ number of entries into the CA of the EPM test, (D) duration of immobility in the TST, and duration of $(E)$ immobility, and $(F)$ swimming in the FST.

with an anxiolytic- and antidepressant-like action. Interestingly, the observed behavioral phenotype was associated to decreased D3R mRNA expression. In contrast, D3R overexpression increased anxiety- and depression-like behaviors as measured in a battery of behavioral tests. The absence of significant modifications in the numbers of line crossings and the number of CA entries (reliable measures of locomotor activity in the OF and EPM tests respectively) indicates that the anxiolytic-like activity, observed following let-7d and D3R overexpression in the hippocampus, did not impair spontaneous locomotor activity. These results indicate that let-7d is a critical regulator for D3R expresssion in mouse hippocampus and we hypothesize consequently that let-7d might participate in the neuropathophysiology of mood disorders through the regulation of D3R genetic expression.

Our initial characterization of the behavioral effects of the functional manipulation of let-7d in the hippocampus showed that let-7d-overexpressing mice display a reduction in their level of anxiety, as evidenced by the increase in time spent in the center of the OF and the increased time spent in the OA of the EPM test. Closer examination suggests that these results were not associated with increased motor activity of these mice, as there was no significant increase in the number of line crossings in the OF test, or in the amount of time spent in the CA of the plus maze. Also, let-7d overexpression decreased the immobility time in both the TST and FST suggesting that it is reasonable to believe that let-7d may play a crucial role in the development of anti-depressant-like behaviors in mice. A substantial literature is emerging supporting miRNAs as differentially expressed in affective disorders. In fact, Mendes-Silva and colleagues conducted a systematic review and found that 
30 miRNAs microRNAs were abnormally expressed in MDD (Mendes-Silva et al., 2016). There is strong evidence that peripheral miRNAs may contribute to the pathophysiology of MDD and antidepressant response. In fact, genetic variants in the promoters of let-7 family have been associated with an increased risk of MDD (Liang et al., 2015). Also, results indicated that, compared to control subjects, let-7d-3p are significantly higher in the serum from the MDD patients compared to healthy controls. The up-regulation of let-7d-3p has been further confirmed in different cohorts of $32 \mathrm{MDD}$ patients (Wan et al., 2015). In addition, it was reported that let-7d-5p expression was altered in MDD patients, and that the bioinformatic prediction of the targeted genes revealed the possible involvement of neural pathways relevant for psychiatric disorders (Maffioletti et al., 2016). Interestingly, Wei and colleagues reported downregulation of let-7 miRNA expression in the prefrontal cortex of a genetic rat model of depression, the Flinders Sensitive Line compared to the control Flinders Resistant Line (Wei et al., 2016). Taken together, these results emphasized the potential role of miRNAs as physiological biomarkers among clinically depressed patients. However, procedural discrepancies in technology used to measure miRNA expression levels, structural differences in miRNA sequences, changes in the severity and forms of depression between patients, and the overall rarity of studies make it difficult to establish any strong, preliminary targets as biomarker potential. Therefore, the ongoing and future studies need to address these methodological and reporting discrepancies in the miRNA research for more consistent candidates associated with MDD. The overexpression of other miRNAs such as miR-17-92, miR-34b also decreased anxiety-like behavior in mice and rats respectively (Jin et al., 2016; Zhu et al., 2017). However, miR-101a$3 \mathrm{p}$-overexpressing adeno-associated viruses (AAVs) in the amygdala increased anxiety-like behavior in the OF and EPM tests of high-novelty-responding (HR) rats (Cohen et al., 2017). Also, miR-155 KO mice exhibited reduced float duration and increased latency to float (Fonken et al., 2016), but, no changes in anxiety-like behavior were observed in miR-132/212 KO mice (Hernandez-Rapp et al., 2015). Differences between all these miRNAs' sequences, target genes, as well as differences between species (rats vs. mice) are likely to contribute to these discrepancies. Furthermore, procedural differences (transgenics vs. viral vectors) may also contribute to these contrasting results.

In an attempt to address one of the mechanisms by which let-7d might decrease anxiety- and depression-like behaviors, we assessed the expression of the D3R in the hippocampus following let-7d overexpression. This target was chosen because previous studies from our laboratory indicated that let-7d manipulation in the nucleus accumbens altered D3R expression levels (Chandrasekar and Dreyer, 2009). Results have shown that reduced D3R levels were associated with an increase of let-7d expression. To the best of our knowledge, we are the first who could demonstrate a negative correlation between levels of D3R and the expression of let$7 \mathrm{~d}$ in the hippocampus. Consequently, we tested the impact of D3R overexpression, using viral vectors, on anxiety- and depression-like behaviors with the hypothesis that, compared to let-7, D3R overexpression would have the opposite effects. As anticipated, the results indicated that D3Roverexpressing mice displayed robust effects predictive of anxiogenic and pro-depressant efficacy without significant effects on locomotor activity, suggesting that targeting the D3R may represent a novel therapeutic avenue for the treatment of some affective disorders.

The potential implication of the D3R in the effects of antidepressants, such as imipramine, amitriptyline, citalopram and mianserin, was suggested by several reports indicating that when administered repeatedly, such drugs enhance the responsiveness of D3R, probably via an increase in the density and/or binding of these receptors (Lammers et al., 2000; Maj et al., 1998). In addition, the D3R is a clinically relevant therapeutic target for anxietyand depression-related behaviors as clinically-used D3Rpreferring agonists, aripiprazole, pramipexole, cariprazine, and ropinirole, have all been shown to have anxiolytic and/or antidepressant effects in humans and animal models (Duric et al., 2017; Rogers et al., 2000; Rogoz et al., 2004; Zarate et al., 2004). However, studies using D3R transgenic mice yielded conflicting results. For instance, D3R KO mice entered the center and the OA significantly more than WT littermates in the OF and EPM tests respectively, but did not differ in CA entries, an index of general spontaneous locomotor activity, with heterozygous mice showing intermediate behavioral changes, suggesting reduced anxiety in mice lacking the D3R (Steiner et al., 1997). However, Chourbaji and co-workers reported D3R KO mice to have regular emotional behavior in a series of selected behavioral paradigms focusing on depressive-like traits (Chourbaji et al., 2008). In contrast, a more recent study revealed that D3R deficiency resulted in anxiety- and depressive-like symptoms that cannot be attributed to motor dysfunction (MoragaAmaro et al., 2014). To circumvent these discrepancies, we strongly believe that, compared to genetically engineered transgenic mice, viral vectors' technology is a relatively rapid and reliable method for in vivo assessment of the D3R in the brain. In addition, the up regulation of D3R, using lentivirus injection in our case, happens at a specific time in the adult mouse (temporal control) and does not include any possibility of leakiness or functional compensation during development, as is most often the case in transgenic mice. Moreover, lentivirus injection provides precise spatial control which, again, is subject to a certain amount of leakiness in the transgenic systems.

Our observations showed that ectopic D3R enhanced the immobility duration in mice in the TST and reduced swimming in the FST. This may suggest that hippocampal D3R overexpression induces a certain neurochemical abnormality which causes the mice to remain immobile in the TST and enter in a state of despair in the FST. Nevertheless, these tests, and particularly the FST, have some disadvantages, such as their unpredictability in uncovering of the effects of a broad spectrum of drugs. In fact, the selective serotonin reuptake inhibitors, a major group of antidepressant drugs, prevent the development of behavioral immobility in the FST (Borsini and Meli, 1988). However, a significant adjustment of the traditional FST procedure confirmed that specific behavioral components of active behaviors in the FST distinguished neurochemically different antidepressant drugs (Lucki, 1997). Indeed, the modified procedure indicated that enhancement of catecholamine neurotransmission may facilitate climbing, whereas enhancement of serotoninergic neurotransmission may mediate swimming. 
(Detke et al., 1995; Lucki, 1997). Regardless, the manifestation of these psychiatric disorders required functional D3R because among dopamine receptor subtypes, the D3R has the highest affinity for dopamine (Sokoloff et al., 1992). One possible explanation is that at least in the hippocampus, permanent D3R overexpression/activation, which might increase receptors' availability, could lead to development of anxiety- and depression-related disorders. Indeed, it is very well established that, in rats, the administration of dopaminergic drugs such as methylphenidate (e.g. Ritalin $₫)$, during pre- and periadolescent development, resulted in the development of psychiatric disorders in adulthood (Bolanos et al., 2003). These results demonstrate the potential dopamine-dependent nature of anxiety- and depressionlike behaviors in rodents and show the possible implication of the D3R. These findings also highlight the need for further research to improve understanding the therapeutic potential of D3R for anxiety, depression, and other related mood disorders.

In conclusion, lentiviral-mediated let-7d overexpression in the hippocampus of adult mice decreases their anxietyand depression-like behaviors and reduces D3R mRNA levels. However, direct D3R overexpression results in the opposite effects. Thus, we hypothesize that increasing the expression of let-7d in the hippocampus produces an anxiolytic- and antidepressant-like effect that is at least partially mediated via the repression of the D3R expression. Overall, these studies point to a novel molecular mechanism that may contribute to individual differences in certain mood disorders. They also add to the growing literature supporting a role for mechanisms once considered to be exclusive to development and differentiation, such as miRNA and epigenetic regulation, in the adult brain and in underlying behavioral phenomena related to psychiatric disorders.

\section{Role of the funding source}

$A B$ was supported by grants from the United Arab Emirates University (No. NP/13/05) and the National Research foundation (No. 31M082). JLD received grants from the Swiss National Science Foundation 3100-059350 and 3100AO-100,686 (JLD). The funders had no further role in study design; in the collection, analysis, and interpretation of data; in the writing of the report; and in the decision to submit the paper for publication.

\section{Disclosure/conflict of interest}

The authors have no financial interests that might be perceived to influence the results, or the discussion reported in this article.

\section{Conflict of interest}

The authors have no financial interests that might be perceived to influence the results, or the discussion reported in this article.

\section{Contributors}

$A B$ designed the study and wrote the protocol. $A B$ and JLD managed the literature searches and analyses. $A B$ undertook the statistical analysis, and AB and JLD wrote the first draft of the manuscript. All authors contributed to and have approved the final manuscript.

\section{Acknowledgments}

The authors would like to acknowledge Christine DeforelPoncet and Vijay Chandrasekar for their technical assistance with the lentiviral vectors preparation. The authors are also grateful to Mohamed Shafiullah and Mahmoud Hag Ali from the Central Animal Facility for their advice on animal care and welfare.

\section{Supplementary materials}

Supplementary material associated with this article can be found

\section{References}

ADAA, 2014. Facts \& Statistics, Anxiety and Depression Association of America. http://www.adaa.org/about-adaa/press-room/ facts-statistics.

Bahi, A., 2017a. Decreased anxiety, voluntary ethanol intake and ethanol-induced CPP acquisition following activation of the metabotropic glutamate receptor 8 "mGluR8". Pharmacol. Biochem. Behav. 155, 32-42.

Bahi, A., 2017b. Environmental enrichment reduces chronic psychosocial stress-induced anxiety and ethanol-related behaviors in mice. Prog. Neuropsychopharmacol. Biol. Psychiatry 77, 65-74.

Bahi, A., Al Mansouri, S., Al Maamari, E., 2016. Nucleus accumbens lentiviral-mediated gain of function of the oxytocin receptor regulates anxiety- and ethanol-related behaviors in adult mice. Physiol. Behav. 164, 249-258.

Bahi, A., Al Mansouri, S., Al Memari, E., Al Ameri, M., Nurulain, S.M., Ojha, S., 2014a. beta-Caryophyllene, a CB2 receptor agonist produces multiple behavioral changes relevant to anxiety and depression in mice. Physiol. Behav. 135, 119-124.

Bahi, A., Boyer, F., Bussard, G., Dreyer, J.L., 2005. Silencing dopamine D3-receptors in the nucleus accumbens shell in vivo induces changes in cocaine-induced hyperlocomotion. Eur. J. Neurosci. 21, 3415-3426.

Bahi, A., Chandrasekar, V., Dreyer, J.L., 2014b. Selective lentiviral-mediated suppression of microRNA124a in the hippocampus evokes antidepressants-like effects in rats. Psychoneuroendocrinology 46, 78-87.

Bahi, A., Dreyer, J.L., 2012. Hippocampus-specific deletion of tissue plasminogen activator "tPA" in adult mice impairs depression- and anxiety-like behaviors. Eur. Neuropsychopharmacol. 22, 672-682.

Bahi, A., Dreyer, J.L., 2014. Lentiviral vector-mediated dopamine d3 receptor modulation in the rat brain impairs alcohol intake and ethanol-induced conditioned place preference. Alcohol Clin. Exp. Res. 38, 2369-2376. 
Bahi, A., Dreyer, J.L., 2017. Viral-mediated overexpression of the Myelin Transcription Factor 1 (MyT1) in the dentate gyrus attenuates anxiety- and ethanol-related behaviors in rats. Psychopharmacology (Berl) 234, 1829-1840.

Bahi, A., Schwed, J.S., Walter, M., Stark, H., Sadek, B., 2014c. Anxiolytic and antidepressant-like activities of the novel and potent non-imidazole histamine $\mathrm{H}(3)$ receptor antagonist ST-1283. Drug Des. Dev. Ther. 8, 627-637.

Beaulieu, J.M., Espinoza, S., Gainetdinov, R.R., 2015. Dopamine receptors - IUPHAR Review 13. Br. J. Pharmacol. 172, 1-23.

Bolanos, C.A., Barrot, M., Berton, O., Wallace-Black, D., Nestler, E.J., 2003. Methylphenidate treatment during pre- and periadolescence alters behavioral responses to emotional stimuli at adulthood. Biol. Psychiatry. 54, 1317-1329.

Borsini, F., Meli, A., 1988. Is the forced swimming test a suitable model for revealing antidepressant activity? Psychopharmacology (Berl) 94, 147-160.

Chandrasekar, V., Dreyer, J.L., 2009. microRNAs miR-124, let-7d and miR-181a regulate cocaine-induced plasticity. Mol. Cell Neurosci. 42, 350-362.

Chandrasekar, V., Dreyer, J.L. , 2011. Regulation of MiR-124, Let-7d, and MiR-181a in the accumbens affects the expression, extinction, and reinstatement of cocaine-induced conditioned place preference. Neuropsychopharmacology 36, 1149-1164.

Cheng, L.C., Tavazoie, M., Doetsch, F., 2005. Stem cells: from epigenetics to microRNAs. Neuron 46, 363-367.

Chourbaji, S., Brandwein, C., Vogt, M.A., Dormann, C., Mueller, R., Drescher, K.U., Gross, G., Gass, P., 2008. Dopamine receptor 3 (D3) knockout mice show regular emotional behaviour. Pharmacol. Res. 58, 302-307.

Cohen, J.L., Jackson, N.L., Ballestas, M.E., Webb, W.M., Lubin, F.D., Clinton, S.M., 2017. Amygdalar expression of the microRNA miR-101a and its target Ezh2 contribute to rodent anxiety-like behaviour. Eur. J. Neurosci. 46, 2241-2252.

Detke, M.J., Rickels, M., Lucki, I., 1995. Active behaviors in the rat forced swimming test differentially produced by serotonergic and noradrenergic antidepressants. Psychopharmacology (Berl) $121,66-72$

Devito, L.M., Kanter, B.R., Eichenbaum, H., 2010. The hippocampus contributes to memory expression during transitive inference in mice. Hippocampus 20, 208-217.

Duric, V., Banasr, M., Franklin, T., Lepack, A., Adham, N., Kiss, B., Gyertyan, I., Duman, R.S., 2017. Cariprazine exhibits anxiolytic and dopamine $\mathrm{d} 3$ receptor-dependent antidepressant effects in the chronic stress model. Int. J. Neuropsychopharmacol. 20, 788-796.

Fonken, L.K., Gaudet, A.D., Gaier, K.R., Nelson, R.J., Popovich, P.G., 2016. MicroRNA-155 deletion reduces anxietyand depressive-like behaviors in mice. Psychoneuroendocrinology $63,362-369$.

Franklin, K.B.J., Paxinos, G., 1996. The Mouse Brain in Stereotaxic Coordinates. Academic Press, New York.

Giridharan, V.V., Thandavarayan, R.A., Fries, G.R., Walss-Bass, C., Barichello, T., Justice, N.J., Reddy, M.K., Quevedo, J., 2016. Newer insights into the role of miRNA a tiny genetic tool in psychiatric disorders: focus on post-traumatic stress disorder. Transl. Psychiatry 6, e954.

Hernandez-Rapp, J., Smith, P.Y., Filali, M., Goupil, C., Planel, E., Magill, S.T., Goodman, R.H., Hebert, S.S., 2015. Memory formation and retention are affected in adult miR-132/212 knockout mice. Behav. Brain Res. 287, 15-26.

Jin, J., Kim, S.N., Liu, X., Zhang, H., Zhang, C., Seo, J.S., Kim, Y., Sun, T., 2016. miR-17-92 cluster regulates adult hippocampal neurogenesis, anxiety, and depression. Cell Rep. 16, 16531663.

Kiebler, M.A., Bassell, G.J., 2006. Neuronal RNA granules: movers and makers. Neuron 51, 685-690.
Lagos-Quintana, M., Rauhut, R., Lendeckel, W., Tuschl, T., 2001. Identification of novel genes coding for small expressed RNAs. Science 294, 853-858.

Lammers, C.H., Diaz, J., Schwartz, J.C., Sokoloff, P., 2000. Selective increase of dopamine D3 receptor gene expression as a common effect of chronic antidepressant treatments. Mol. Psychiatry 5, 378-388.

Leggio, G.M., Bucolo, C., Platania, C.B., Salomone, S., Drago, F., 2016. Current drug treatments targeting dopamine D3 receptor. Pharmacol. Ther. 165, 164-177.

Liang, Y., Zhao, G., Sun, R., Mao, Y., Li, G., Chen, X., Gao, L., $\mathrm{Hu}, \mathrm{Z}$., 2015. Genetic variants in the promoters of let-7 family are associated with an increased risk of major depressive disorder. J. Affect. Disord. 183, 295-299.

Liu, C., Zhao, X., 2009. MicroRNAs in adult and embryonic neurogenesis. Neuromol. Med. 11, 141-152.

Lucki, I., 1997. The forced swimming test as a model for core and component behavioral effects of antidepressant drugs. Behav. Pharmacol. 8, 523-532.

Luo, T., Yin, S., Shi, R., Xu, C., Wang, Y., Cai, J., Yue, Y., Wu, A. 2015. miRNA expression profile and involvement of Let-7d-APP in aged rats with isoflurane-induced learning and memory impairment. PLoS One 10, e0119336.

Maffioletti, E., Cattaneo, A., Rosso, G., Maina, G., Maj, C., Gennarelli, M., Tardito, D., Bocchio-Chiavetto, L., 2016. Peripheral whole blood microRNA alterations in major depression and bipolar disorder. J. Affect. Disord. 200, 250-258.

Maj, J., Dziedzicka-Wasylewska, M., Rogoz, R., Rogoz, Z., 1998. Effect of antidepressant drugs administered repeatedly on the dopamine D3 receptors in the rat brain. Eur. J. Pharmacol. 351, 31-37.

Mendes-Silva, A.P., Pereira, K.S., Tolentino-Araujo, G.T., Nicolau Ede, S., Silva-Ferreira, C.M., Teixeira, A.L., Diniz, B.S., 2016. Shared biologic pathways between alzheimer disease and major depression: a systematic review of microRNA expression studies. Am. J. Geriatr. Psychiatry 24, 903-912.

Moraga-Amaro, R., Gonzalez, H., Pacheco, R., Stehberg, J., 2014. Dopamine receptor D3 deficiency results in chronic depression and anxiety. Behav. Brain Res. 274, 186-193.

Rogers, D.C., Costall, B., Domeney, A.M., Gerrard, P.A., Greener, M., Kelly, M.E., Hagan, J.J., Hunter, A.J., 2000. Anxiolytic profile of ropinirole in the rat, mouse and common marmoset. Psychopharmacology (Berl) 151, 91-97.

Rogoz, Z., Skuza, G., Kllodzinska, A., 2004. Anxiolytic- and antidepressant-like effects of 7-OH-DPAT, preferential dopamine D3 receptor agonist, in rats. Pol. J. Pharmacol. 56, 519526.

Roush, S., Slack, F.J., 2008. The let-7 family of microRNAs. Trends Cell Biol. 18, 505-516.

Shu, S.Y., Qing, D., Wang, B., Zeng, Q.Y., Chen, Y.C., Jin, Y., Zeng, C.C., Bao, R., 2013. Comparison of microRNA expression in hippocampus and the marginal division (MrD) of the neostriatum in rats. J. Biomed. Sci. 20, 9.

Sokoloff, P., Giros, B., Martres, M.P., Bouthenet, M.L., Schwartz, J.C., 1990. Molecular cloning and characterization of a novel dopamine receptor (D3) as a target for neuroleptics. Nature 347, 146-151.

Sokoloff, P., Martres, M.P., Giros, B., Bouthenet, M.L., Schwartz, J.C., 1992. The third dopamine receptor (D3) as a novel target for antipsychotics. Biochem. Pharmacol. 43, 659-666.

Steiner, H., Fuchs, S., Accili, D. , 1997. D3 dopamine receptor-deficient mouse: evidence for reduced anxiety. Physiol. Behav. 63, 137-141.

Suarez-Gomez, M., Alejandre-Duran, E., Ruiz-Rubio, M., 2011. [MicroRNAs in bipolar disorder: diagnostic and therapeutic applications]. Rev. Neurol. 53, 91-98. 
Tabares-Seisdedos, R., Rubenstein, J.L., 2009. Chromosome 8p as a potential hub for developmental neuropsychiatric disorders: implications for schizophrenia, autism and cancer. Mol. Psychiatry 14, 563-589.

Uchida, S., Nishida, A., Hara, K., Kamemoto, T., Suetsugi, M., Fujimoto, M., Watanuki, T., Wakabayashi, Y., Otsuki, K., McEwen, B.S., Watanabe, Y., 2008. Characterization of the vulnerability to repeated stress in Fischer 344 rats: possible involvement of microRNA-mediated down-regulation of the glucocorticoid receptor. Eur. J. Neurosci. 27, 2250-2261.

Wan, Y., Liu, Y., Wang, X., Wu, J., Liu, K., Zhou, J., Liu, L., Zhang, C., 2015. Identification of differential microRNAs in cerebrospinal fluid and serum of patients with major depressive disorder. PLoS One 10, e0121975.

Wei, Y.B., Liu, J.J., Villaescusa, J.C., Aberg, E., Brene, S., Wegener, G., Mathe, A.A., Lavebratt, C., 2016. Elevation of Il6 is associated with disturbed let-7 biogenesis in a genetic model of depression. Transl. Psychiatry 6, e869.
Wu, L., Zhao, Q., Zhu, X., Peng, M., Jia, C., Wu, W., Zheng, J., Wu, X.Z., 2010. A novel function of microRNA let-7d in regulation of galectin-3 expression in attention deficit hyperactivity disorder rat brain. Brain Pathol. 20, 1042-1054.

Zarate Jr., C.A., Payne, J.L., Singh, J., Quiroz, J.A., Luckenbaugh, D.A., Denicoff, K.D., Charney, D.S., Manji, H.K., 2004. Pramipexole for bipolar II depression: a placebo-controlled proof of concept study. Biol. Psychiatry 56, 54-60.

Zhao, C., Sun, G., Ye, P., Li, S., Shi, Y., 2013. MicroRNA let-7d regulates the TLX/microRNA-9 cascade to control neural cell fate and neurogenesis. Sci. Rep. 3, 1329.

Zhu, J., Chen, Z., Tian, J., Meng, Z., Ju, M., Wu, G., Tian, Z., 2017. miR-34b attenuates trauma-induced anxiety-like behavior by targeting CRHR1. Int. J. Mol. Med. 40, 90-100. 